\title{
Three dimensional periodic orbits in exterior mean motion resonances with Neptune
}

\author{
T. A. Kotoulas and G. Voyatzis \\ University of Thessaloniki, Department of Physics, 54124 Thessaloniki, Greece \\ e-mail: tkoto@skiathos.physics.auth.gr \\ Received 3 March 2005 / Accepted 7 June 2005

\begin{abstract}
In the framework of the three dimensional restricted three-body problem we study the dynamics of the exterior mean motion resonances with Neptune. The basis of our study is the computation of periodic orbits and their linear stability. The position and stability of periodic orbits critically determine the phase space topology. Stable periodic orbits are centers of regular librations where resonant trapping of minor bodies can take place. The periodic orbits are continued analytically from the planar model to the three-dimensional one forming families, which, in most cases, extend up to high inclination values and pass to the regime of retrograde motion. A classification of the families, according to their continuation and termination, is given. The computations show that resonant librating motion is present up to high inclination values and for particular eccentricity domains. When the ellipticity of the Neptune's orbit is taken into account, periodic orbits are found only for very high inclination values and all of them are unstable.
\end{abstract}

Key words. celestial mechanics - Kuiper belt - minor planets, asteroids - methods: numerical

\section{Introduction}

The restricted three body problem (RTBP) is the basic model for studying the dynamics of small bodies in our Solar system. Particularly, it has been applied widely to understand the structure of the Kirkwood gaps in the asteroid belt and the dynamics of the Edgeworth-Kuiper belt. The dynamics of these belts of minor bodies is strongly associated with resonant motion. Resonances are responsible for either unstable motion, which ejects the bodies far from their elliptic motion, or for stable motion, which protects the small bodies from strong interactions with the associated planet and provides the conditions for long-term regular evolution.

Particularly, the dynamics of trans-Neptunian objects (TNOs) that form the EKB is of significant interest in the last decade since more than 800 objects have been observed up to now (Minor Planet Center, http:// cfa-www.harvard.edu/iau/mpc.html). Both observations and dynamical studies show that the EKB has an interesting structure. By understanding its dynamics we may obtain new mechanisms that played a role in the current formation of our Solar system (see the review papers of Morbidelli 1999; and Morbidelli et al. 2003, and references therein). One of the main problems in Kuiper-belt studies is the explanation of highinclination orbits, which are possessed by a significant portion of TNOs. The numerical simulations indicate the possibility of long-term stability of high inclined orbits up to $30^{\circ}$ (Kuchner et al. 2002; Gomes 2003). Recent observations discovered TNOs in orbits with inclinations up to $40^{\circ}$.
In the planar circular RTBP, regular resonant motion is described by librations with respect to the resonant angle $\sigma=$ $p \lambda^{\prime}-q \lambda-(p-q) \varpi$, where $p / q$ denotes the external resonance $(p<q)$ (Malhotra 1996; Celleti et al. 2002). Stable librations take place around linearly stable periodic orbits which are isolated in phase space. The method of Poincaré section can be applied providing a comprehensive picture of the phase space structure. The existence of stable periodic orbits, which correspond to elliptic fixed points in Poincaré sections, confirms the existence of regions of stable motion around them. Additionally, these regions are potentials for resonance trapping in more complicated models that take into account migration of planets and drag (Beaugé \& Ferraz-Mello 1994; Hahn \& Malhotra 1999; Yu \& Tremaine 2001; Gomes et al. 2004). Therefore, the main features of the dynamics of the basic RTBP model are relative to those shown in more realistic models.

For inclined orbits, the spatial or three-dimensional (3D) RTBP should be considered as the basic model. In this case the phase space is six-dimensional and the Poincaré section, which is four dimensional, is not convenient and adequate, as in the planar model, to describe the phase space structure. However, even in this case the periodic orbits are of great importance for understanding the topology of phase space and localizing stable motion (Hadjidemetriou 1988). Except for the dynamics of minor bodies in our Solar System, other applications of resonant periodic orbits of the RTBP are found also in the dynamics of extrasolar systems (Varadi 1999; Haghighipour et al. 2003). 
The aim of the present work is a systematic study of three dimensional (3D) periodic orbits of the RTBP associated with exterior mean motion resonances. We localize all the families of three-dimensional periodic orbits of the system that bifurcate from the planar problem. Our study covers the whole eccentricity interval $(0<e<1)$ and inclination values $\left(0<i<180^{\circ}\right)$, i.e. retrograde motion is also included. Particularly, we study resonances of the form $p /(p+q)$ of first, second and third order ( $q$ denotes the resonance order). In Sect. 2 we present the methodology of our computations and discuss the structure of the families of 3D periodic orbits. Section 3 presents our computations about 3D resonant periodic orbits and their families. The dynamics associated with the stable periodic orbits and the structure of the phase space is discussed. In Sect. 4, we present families of periodic orbits in the 3D elliptic RTBP and we discuss the effect of the ellipticity of the orbit of Neptune on the evolution of orbits.

The model under consideration is the three-dimensional RTBP (Szebehely 1967). In order to make our results relevant to the basic dynamics of Edgeworth-Kuiper belt, we take as primaries the Sun of mass $1-\mu$ and the Neptune of mass $\mu=5.178 \times 10^{-5}$. In the normalized units the gravitational constant is equal to one. In the inertial orthogonal frame $O X Y Z$ the motion of Neptune is either circular or elliptic on the plane $O X Y$ round the center $O$ with eccentricity $e^{\prime}$, semimajor axis $a^{\prime}=1$ and period $T^{\prime}=2 \pi$. In the rotating orthogonal frame of reference $O x y z$, where the Sun and the Neptune define the $O x$-axis, the motion of the small body is described by the Lagrangian

$L=\frac{1}{2}\left[(\dot{x}-\dot{\theta} y)^{2}+(\dot{y}+\dot{\theta} x)^{2}+\dot{z}^{2}\right]+\frac{1-\mu}{r_{1}}+\frac{\mu}{r_{2}}$,

where $r_{1}^{2}=(x+\mu r)^{2}+y^{2}+z^{2}, r_{2}^{2}=(x-1+\mu r)^{2}+y^{2}+z^{2}$, $r$ is the distance between the primaries and $\theta$ the angle between the axes $O x$ and $O X$. For the circular case it is $r=1, \dot{\theta}=1$ and there exists the Jacobi constant $h$

$h=\frac{1}{2}\left[\dot{x}^{2}+\dot{y}^{2}+\dot{z}^{2}-\left(x^{2}+y^{2}\right)\right]-\frac{1-\mu}{r_{1}}-\frac{\mu}{r_{2}}$.

\section{Periodicity conditions and family classification in the 3D circular problem}

Three dimensional symmetric periodic orbits of the circular RTBP have been studied by several authors (Henon 1969; Zagouras \& Markellos 1977; Michalodimitrakis 1979; Broucke 2001; Papadakis 2004). These studies refer to families of $3 \mathrm{D}$ periodic orbits associated with the dynamics near the Lagrangian equilibrium points. In the study of mean motion resonances, two kinds of periodic orbits, indicated by Poincaré (1892), are considered:

Families of circular orbits: the periodic orbits correspond to nearly circular orbits for the small body i.e. the corresponding initial eccentricity value $e_{0}$ is almost zero. The period $T$, and subsequently the resonance $n / n^{\prime}=T / T^{\prime}$, varies along the family.

Families of elliptic orbits: the periodic orbits correspond to almost elliptic orbits for the small body and they are resonant.
The eccentricity $e_{0}$ increases along the family but the ratio $n / n^{\prime}$ is almost constant and rational $n / n^{\prime} \approx p / q, p, q \in Z$.

For both kinds of periodic orbits the initial inclination $\left(i_{0}\right)$ starts from zero and varies along the family. Thus, the 3D periodic orbits originate from the planar periodic orbits in the $O x y$ plane. The planar orbits, which are continued as periodic orbits in the 3D space $O x y z$, are critical with respect to the vertical stability (Henon 1973; Michalodimitrakis 1979). Analytic continuation of these orbits can take place in two ways giving rise to the following families:

Families $F$ : they consist of symmetric periodic orbits with respect to $x z$-plane. In this case a periodic orbit can be defined by the initial conditions $x(0)=x_{0}, y(0)=0, z(0)=z_{0}, \dot{x}(0)=0$, $\dot{y}(0)=\dot{y}_{0}, \dot{z}(0)=0$ and the periodicity conditions are:

$y(0)=y(T / 2)=0, \dot{x}(0)=\dot{x}(T / 2)=0, \dot{z}(0)=\dot{z}(T / 2)=0,(3)$

where $T$ is the period of the orbit. We can represent such a periodic orbit as a point in $3 \mathrm{D}$ space $x_{0}-\dot{y}_{0}-z_{0}$ or $x_{0}-z_{0}-h$, where $h$ is the corresponding Jacobi constant. By varying the value of $h$ we get a monoparametric family of periodic orbits, which is represented by a smooth curve in the space $x_{0}-z_{0}-h$. From the geometry of the orbit it can be seen that at $t=0$ the longitude of the ascending node $(\Omega)$ is equal to $\Omega=\pi / 2$ and the small body is at perihelion $(\varpi=0)$ or aphelion $(\varpi=\pi)$.

Families $G$ : the periodic orbits are symmetric with respect to $x$-axis. In this case a periodic orbit can be defined by the initial conditions $x(0)=x_{0}, y(0)=0, z(0)=0, \dot{x}(0)=0$, $\dot{y}(0)=\dot{y}_{0}, \dot{z}(0)=\dot{z}_{0}$ and the periodicity conditions are:

$y(0)=y(T / 2)=0, \dot{x}(0)=\dot{x}(T / 2)=0, \quad z(0)=z(T / 2)=0$.

We can represent such a periodic orbit as a point in 3D space $x_{0}-\dot{y}_{0}-\dot{z}_{0}$ or $x_{0}-\dot{z}_{0}-h$, where $h$ is the corresponding Jacobi constant. By varying the value of $h$ we get a monoparametric family of periodic orbits which is represented by a smooth curve in the space $x_{0}-\dot{z}_{0}-h$. From the geometry of the orbit it can be seen that at $t=0$ the longitude of the ascending node $(\Omega)$ is equal to $\Omega=0$ and the small body is at perihelion $(\varpi=0)$ or aphelion $(\varpi=\pi)$.

Since the origin of the families is known, their continuation is accomplished by the method of differential corrections (Broucke 1969; Varadi 1999) up to a sufficient numerical approximation of the periodicity conditions. In the present work the periodicity conditions are fulfilled with accuracy $10^{-13}$. The linear stability of the periodic orbits is determined by the corresponding stability indices $b_{1}$ and $b_{2}$ provided by the numerical solutions of the variational equations (Szebehely 1967; Michalodimitrakis 1979).

The analytical continuation of a family $F$ or $G$ from a vertical critical planar orbit is proved only for small inclination values. However, in most cases studied in the literature and in the present study too, the families continue smoothly up to high inclination values and may be corrupted only due to collisions. 


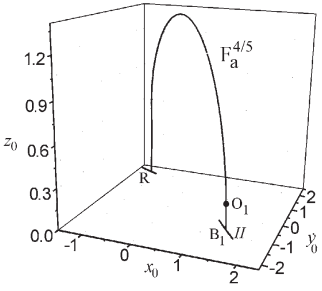

(a)

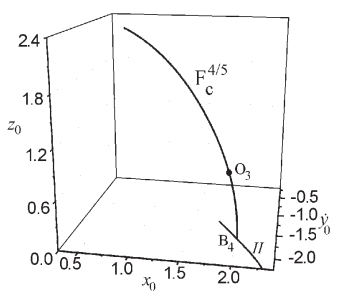

(c)

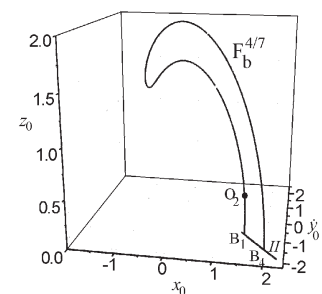

(b)

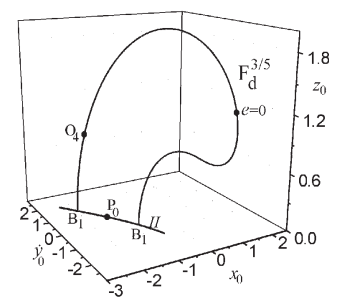

(d)
Fig. 1. Four representative families $F$ of type a)-d) of symmetric 3D periodic orbits in the $x_{0}-\dot{y}_{0}-z_{0}$ space. The points $B_{i}$ indicate the vertical critical orbits belonging to the family II of the planar system (see Sect. 3). $R$ denotes the family of retrograde periodic orbits.

By taken into account this fact we introduce a new classification of the families according to their termination point. Four different types of families are described in the following:

- Type $a$ : this family of 3D periodic orbits starts from a vertical critical orbit of the planar circular model and terminates at a retrograde planar periodic orbit. Thus the inclination $i_{0}$ along the family takes values in the domain $\left(0,180^{\circ}\right)$. An example of such a family in the $4 / 5$ resonance is shown in Fig. 1a.

- Type $b$ : such families start from a vertical critical planar orbit $B$ and terminate at another one $B^{\prime}$. Our numerical results suggest that $B$ and $B^{\prime}$ belong to the same family of periodic orbits of the planar circular problem. An example is given in Fig. 1b.

- Type c: families of this type terminate at a collision orbit with Sun. In most cases such families start from vertical critical orbits with high eccentricity values and are stable (see Sect. 3). An example is given in Fig. 1c.

- Type $d$ : these families start and terminate at the same vertical critical orbit. In Fig. 1d we present a family of this type obtained in the resonance $3 / 5$. Although, the family seems to start and terminate at different points on the family II of the planar orbits, in fact these points correspond to the same orbit. The point $P_{0}$ on the family II separates the family in two equivalent sections that include the same orbits, but the periodic orbits are represented by different initial conditions $\left(x_{0}, \dot{y}_{0}\right)$ in each section (see also Fig. 4 ).

In the following we adopt the family notation $F_{t}^{p / q}$ or $G_{t}^{p / q}$, where $t$ denotes the type (a), (b), (c) or (d) according to the above classification and $p / q$ denotes the resonance. Some typical examples of periodic orbits, denoted as $O_{i}$ in the families of Fig. 1, are presented in Fig. 2 in the rotating frame Oxyz.
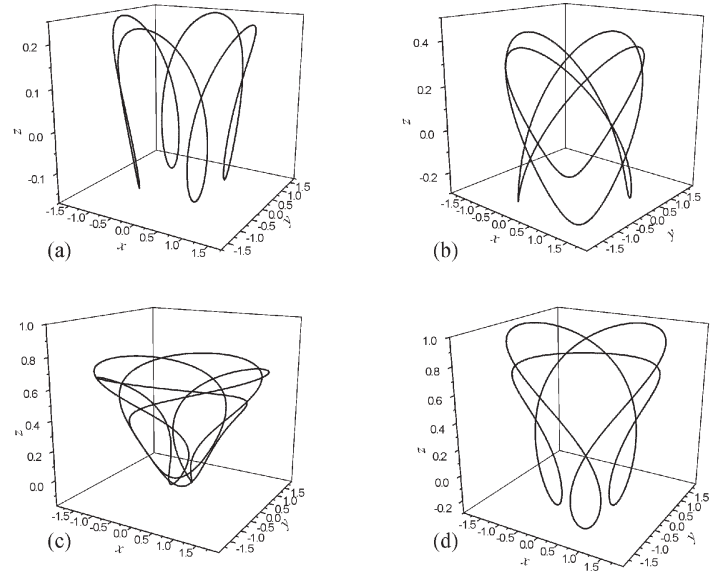

Fig. 2. Four representative 3D symmetric periodic orbit of type $F$ which belong to the families shown in Fig. 1 These are: a) $\mathrm{O}_{1}$, b) $\mathrm{O}_{2}$, c) $\mathrm{O}_{3}$ and d) $\mathrm{O}_{4}$ correspondingly.

\section{Families of 3D orbits in the circular RTBP}

We mentioned in the previous section that the families of three dimensional periodic orbits originate from the vertically critical orbits (VCOs) of the planar circular problem. For the EKB resonances, the planar circular problem has been extensively studied (Malhotra 1996; Kotoulas \& Hadjidemetriou 2002; Voyatzis \& Kotoulas 2005b). For all resonances (particularly of first, second and third order), the circular planar RTBP admits two families of symmetric periodic orbits called family $I$ and family $I I$ which bifurcate from the family of circular orbits. In family I the small body is at perihelion at $t=0$, while in family II the small body is at aphelion at $t=0$ (for more details, see Voyatzis \& Kotoulas 2005b). In Kotoulas $\&$ Voyatzis (2005a) the position of VCOs are given for all first, second and third order resonances located between 32 and $45 \mathrm{AU}$. For the first and third order resonances VCOs exist in family II exclusively.

The family of circular orbits $C$ in the planar case is not continued near the first order resonances for $\mu>0$. So, it is not expected to have $3 \mathrm{D}$ circular periodic orbits in this case. For the second order resonances VCOs exist along the families I and II of the planar circular model. Moreover, the family of circular orbits $C$ in the planar case is continued near the second order resonances for $\mu>0$ with an unstable branch of circular periodic orbits. From this branch two families of symmetric 3D periodic orbits are generated, one of symmetry F and one of symmetry G (see also Sects. 3.2 and 3.3). In the case of third order resonances, VCOs exist along the families II similarly to the first order resonances. Additionally, the family of planar circular orbits $C$ is continued for $\mu>0$ and includes VCOs too. In Table 1 we present the corresponding Jacobi values of circular VCOs.

In Kotoulas \& Hadjidemetriou (2002) the first order resonances $1 / 2,2 / 3$ and $3 / 4$ were studied and the corresponding families of $3 \mathrm{D}$ periodic orbits were given. According to the classification introduced in Sect. 2, they determined the families: $F_{c}^{1 / 2}, G_{a}^{1 / 2}, F_{c}^{2 / 3}, G_{b}^{2 / 3}$ and $F_{a 1}^{3 / 4}, F_{a 2}^{3 / 4}, G_{b}^{3 / 4}, F_{c}^{3 / 4}$. In the following, we study the families of three dimensional periodic 


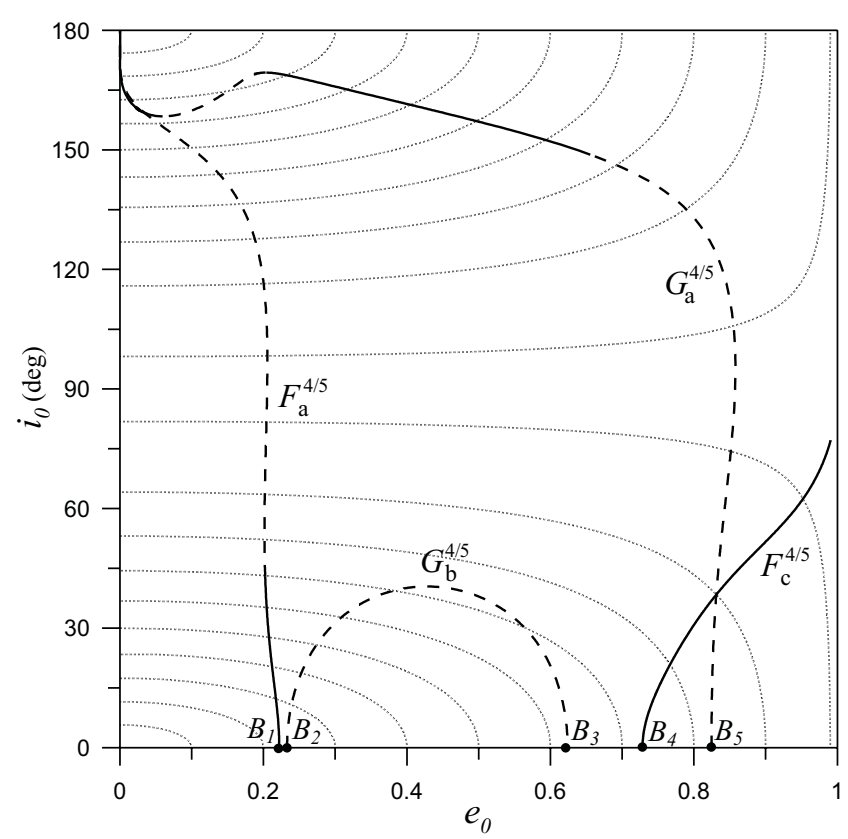

Fig. 3. Families of $4 / 5$ resonant $3 D$ symmetric periodic orbits in the circular RTBP. Solid segments denote linear stability and dashed lines stand for linear instability. The thin dotted lines denote the curves of $z$-argument of the angular momentum related to the Kozai dynamics.

Table 1. The Jacobi value of the vertically critical circular orbits $(e \approx 0)$. From each of these orbits two families, one of symmetry $\mathrm{F}$ and one of symmetry G, are generated. The symbol "S" (stable) or "U" (unstable) denote the linear stability of the orbits in the generated families of 3D periodic orbits.

\begin{tabular}{cc|cc}
\hline \hline Res. & $h$ and type & Res. & $h$ and type \\
\hline $3 / 5$ & $-1.541 \mathrm{FU}, \mathrm{GU}$ & $4 / 7$ & $-1.549 \mathrm{FU}, \mathrm{GS}$ \\
$5 / 7$ & $-1.518 \mathrm{FU}, \mathrm{GU}$ & $5 / 8$ & $-1.535 \mathrm{FU}, \mathrm{GS}$ \\
$7 / 9$ & $-1.510 \mathrm{FU}, \mathrm{GU}$ & $7 / 10$ & $-1.520 \mathrm{FU}, \mathrm{GS}$ \\
\hline
\end{tabular}

orbits located in resonances of different order. We present analytically the results obtained for the the $4 / 5,3 / 5$ and $4 / 7$ resonances. From a physical point of view, it is more convenient to present the families in the projection plane $e_{0}-i_{0}$. The family segments that consist of linearly stable orbits are indicated by a solid line, while unstable segments are indicated by a dashed line. The results of other resonant cases are summarized in Sect. 3.4.

\subsection{The $4 / 5$ resonance}

There are five VCOs which are starting points for continuing families of $3 \mathrm{D}$ periodic orbits. They belong to the family $\mathrm{II}_{4 / 5}$ of the planar circular problem and are the following ones: $B_{1}\left(e_{0}=0.22\right), B_{2}\left(e_{0}=0.23\right), B_{3}\left(e_{0}=0.62\right), B_{4}\left(e_{0}=0.73\right)$ and $B_{5}\left(e_{0}=0.83\right)$. The computed families are shown in Fig. 3.

The family $F_{a}^{4 / 5}$ originates from the point $B_{1}$ and consists of stable orbits. The stability is preserved up to $i_{0}=43^{\circ}$. The eccentricity $e_{0}$ of periodic orbits is almost constant up to $i_{0}=120^{\circ}$. Then $e_{0}$ starts to decrease and the family terminates at a circular planar retrograde orbit. From the point $B_{2}$,

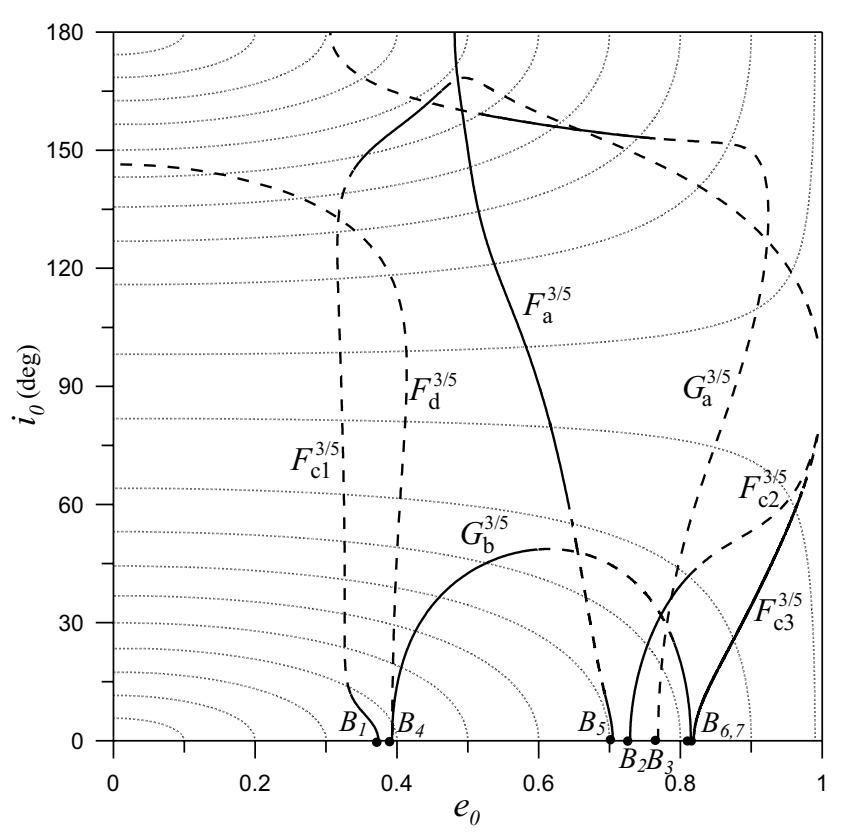

Fig. 4. Resonance 3/5: families of periodic orbits in 3D circular RTBP.

we get the family $G_{b}^{4 / 5}$, which terminates at the bifurcation point $B_{3}$. All orbits are unstable and the maximum inclination value reached is about $40^{\circ}$. The family $F_{c}^{4 / 5}$, which starts at the highly eccentric point $B_{4}$, is stable and terminates at a collision with Sun $(e \approx 1)$ at $i_{0} \approx 90^{\circ}$. Finally, the family $G_{a}^{4 / 5}$, which originates at $B_{5}$, is whole unstable for direct motion (i.e. $i_{0}<90^{\circ}$ ). Similarly to the family $F_{a}^{4 / 5}$, the eccentricity along $G_{a}^{4 / 5}$ remains almost constant up to $i_{0}=120^{\circ}$, then it decreases rapidly and the family terminates at a circular retrograde orbit. Two segments of stable orbits are obtained for $i_{0} \geq 150^{\circ}$.

Conclusively, stability regions with $4 / 5$ resonant librating motion can be found for $e \approx 0.2$ and for $i<43^{\circ}$. The family $F_{c}^{4 / 5}$, though it is stable, can not be taken into account for concluding about the basic dynamics of the Edgeworth-Kuiper belt. Its orbits are of high eccentricity and, subsequently, the minor body strongly interacts with the other bodies in the inner Solar system.

The critical periodic orbits, where the stability type changes, are bifurcation points for families with either symmetric periodic orbits of larger multiplicity (i.e. larger period) or asymmetric orbits. The latter case and for the planar problem is possible only for resonances of the form $1 / q$. In this work we do not compute the above mentioned types of periodic orbits.

\subsection{The $3 / 5$ resonance}

In higher order resonances, the number of VCOs is larger with respect to the first order resonances. In the 3/5 resonance, we found eight VCOs in the corresponding planar families I, II and $C$. In family I there exist three VCOs at the points $B_{1}\left(e_{0}=0.37\right), B_{2}\left(e_{0}=0.73\right)$, and $B_{3}\left(e_{0}=0.77\right)$; in family II four VCOs are found at the points $B_{4}\left(e_{0}=0.39\right)$, $B_{5}\left(e_{0}=0.70\right), B_{6}\left(e_{0}=0.81\right)$ and $B_{7}\left(e_{0}=0.82\right)$. Additionally, the family of circular orbits in the planar problem includes 

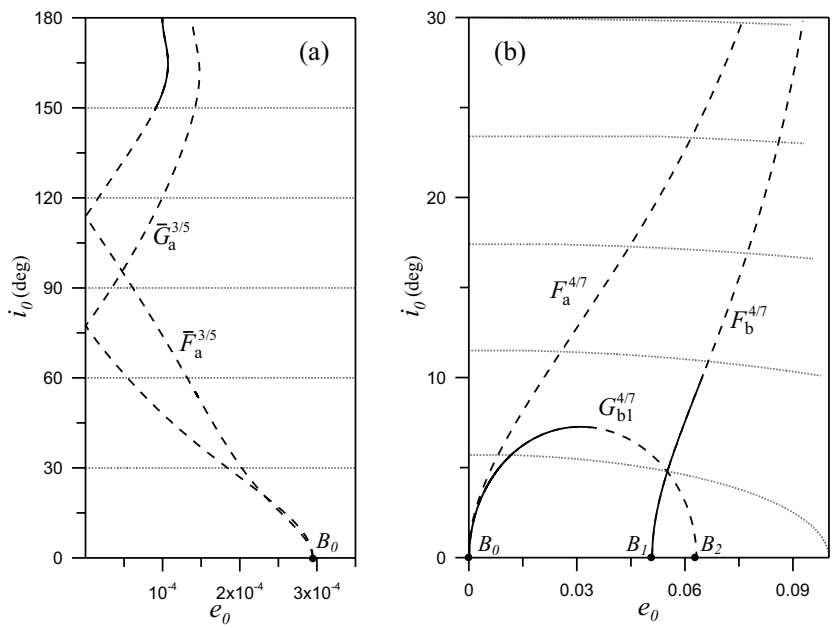

Fig. 5. a) Resonance 3/5: families of periodic orbits in $3 \mathrm{D}$ circular RTBP which originate from the unstable branch of the family $C$. b) Resonance 4/7: families of periodic orbits in 3D circular RTBP which originate from the stable branch of the family $C$ of circular orbits and from low-eccentric VCOs.

one $\mathrm{VCO}$, denoted by $B_{0}$ at $e_{0} \approx 0.0003$. The generating families are shown in Fig. 4.

The VCO $B_{0}$ of the circular planar family $C$ is a bifurcation point of the two families, $\bar{F}_{a}^{3 / 5}$ and $\bar{G}_{a}^{3 / 5}$, shown in Fig. 5a. Along the whole inclination interval $\left(0,180^{\circ}\right)$, the eccentricity remains small. At a particular value of the inclination (see Fig. 5a) the eccentricity becomes exactly zero and at this point the orbit changes its longitude of pericenter from $\varpi=0$ to $\varpi=180^{\circ}$ in family $\bar{F}_{a}^{3 / 5}$ and inversely in family $\bar{G}_{a}^{3 / 5}$. The family $\bar{G}_{a}^{3 / 5}$ is unstable while the family $\bar{F}_{a}^{3 / 5}$ has a stable segment for $i_{0}>140^{\circ}$.

Except for the VCOs in the circular family of the planar problem, the rest are located to relatively large eccentricity values. The first family $F_{c 1}^{3 / 5}$, which originates at $B_{1}$, terminates at a collision with Sun at about $i=90^{\circ}$ (Fig. 4). The families $F_{c 2}^{3 / 5}$ and $F_{c 3}^{3 / 5}$, which start from the VCOs $B_{4}$ and $B_{7}$, respectively, also terminate with a collision for the same inclination value. The families $F_{a}^{3 / 5}$ and $G_{a}^{3 / 5}$ which start from the VCOs $B_{5}$ and $B_{3}$, respectively, show a normal evolution and terminate at retrograde eccentric planar orbits. Up to the accuracy of the calculations, the VCO $B_{2}$ seems to be a bifurcation point for the families $G_{b}^{3 / 5}$ and $F_{d}^{3 / 5}$. The family $G_{b}^{3 / 5}$ extends up to $i \approx 45^{\circ}$ and terminates at the VCO $B_{6}$. The family $F_{d}^{3 / 5}$ extends up to $i=145^{\circ}$ where the orbits become circular. At this point the continuation of the family provides the same periodic orbits and the family terminates at the point $B_{2}$. The structure of families of type $d$ has been discussed in Sect. 2 .

All families, except for the circular ones, $G_{a}^{3 / 5}$ and $F_{d}^{3 / 5}$, start with stable orbits. Also, segments of stable orbits are obtained for highly inclined direct motion $\left(i_{0}>60^{\circ}\right)$ in family $F_{a}^{3 / 5}$. For $0 \leq e \leq 0.3$ there is lack of stable periodic orbits and, subsequently, no regular resonant librating motion can be found in the dynamics of the particular resonance.

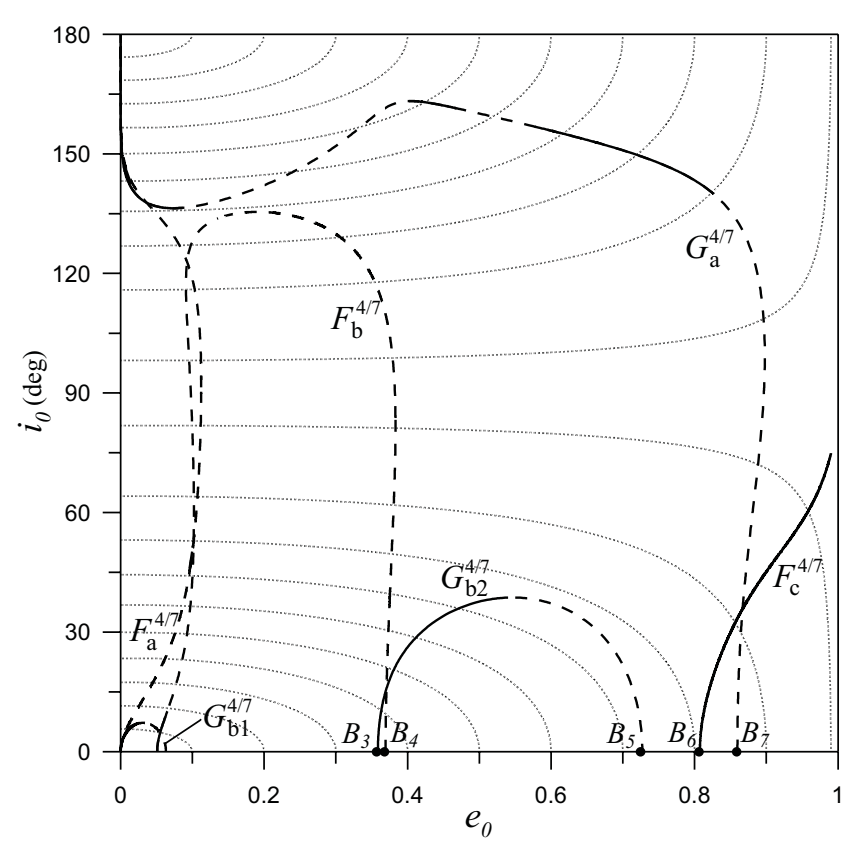

Fig. 6. Families of $4 / 7$ resonant periodic orbits in the 3-D circular RTBP.

\subsection{The $4 / 7$ resonance}

Similarly to the first order resonance $4 / 5$, VCOs are found on the family $\mathrm{II}_{4 / 7}$ of the planar circular problem. These VCOs are the following: $B_{1}(e=0.05), B_{2}(e=0.06), B_{3}(e=0.36)$, $B_{4}(e=0.37), B_{5}(e=0.73), B_{6}(e=0.81)$ and $B_{7}(e=0.86)$. Additionally, one $\operatorname{VCO}\left(B_{0}\right)$ is found in the circular family $C$. The generated families of $3 \mathrm{D}$ periodic orbits, starting from low eccentricity values, are shown in Fig. 5b. A global view is given in Fig. 6. The VCO $B_{0}$ is a bifurcation point providing the formation of two families, $F_{a}^{4 / 7}$ and $G_{b 1}^{4 / 7}$. Also, as in the case of $4 / 5$ resonance, the families of type " $a$ " terminate at a circular retrograde orbit of the circular problem.

\subsection{Phase space topology and stable periodic orbits in other resonances}

The periodic orbits of the 3D circular RTBP are associated with the Kozai resonance. Namely, the stable periodic orbits are centers of $\omega$-librating motion (for a detailed description of the dynamics of Kozai resonance see Morbidelli 2002). The orbits of families $F$ correspond to $\omega=\pi / 2$ or $-\pi / 2$ (and $\Omega=\pi / 2$ or $-\pi / 2$, respectively) and the orbits of families $G$ correspond to $\omega=0$ or $\pi$ (and $\Omega=\pi$ or 0 , respectively). If a periodic orbit is stable, then regular motion exists in a phase space domain near the periodic orbit, which is characterized by the libration of $\omega$ around the above mentioned values (see also Fig. 9a). Also, a strong coupling between the evolution of eccentricity and inclination holds, which is described by the relation $h_{z}=\sqrt{a\left(1-e^{2}\right)} \cos i \approx$ const., where $h_{z}$ is the $z$-component of angular momentum. The contours $h_{z}(e, i)=$ const. are included in Figs. 3-5. In Fig. 7a we illustrate the phase space structure around the periodic orbit of family $F_{a}^{4 / 5}$ at $e=0.219$ and $i=10^{\circ}$ by presenting the projection of the four dimensional 

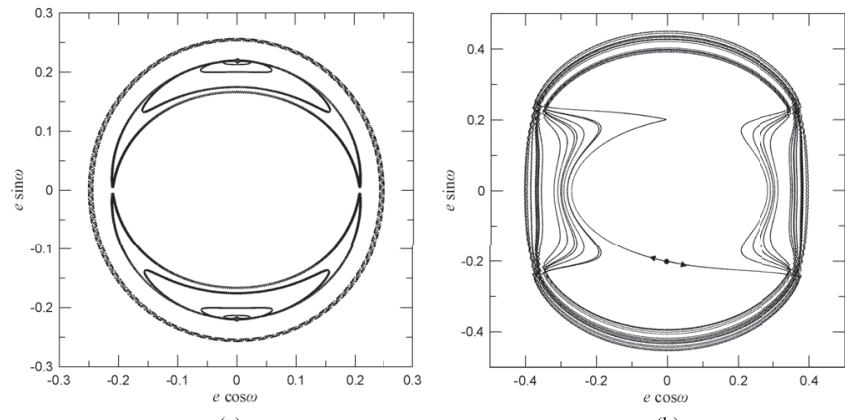

(a)

(b)

Fig. 7. Projections of the 4-D Poincaré section in the domain around a periodic orbit (the corresponding fixed points are indicated by the bold dots). a) Section of orbits in the region of a stable periodic orbit of family $F_{a}^{4 / 5}\left(i_{0}=10^{\circ}\right)$. b) Poincaré section of two trajectories that start close to an unstable periodic orbit of family $F_{a}^{4 / 5}\left(i_{0}=50^{\circ}\right)$. The arrows indicate the initial directions of the evolution of the two trajectories.

Table 2. Potential regions including centers for librating resonant motion for $e<0.4$ and $i>0$ in the circular RTBP.

\begin{tabular}{cccc}
\hline \hline Resonance & $a_{\text {res }}(\mathrm{AU})$ & $\left(e_{\min }, e_{\max }\right)$ & $i_{\max }\left({ }^{\circ}\right)$ \\
\hline $2 / 3$ & 39.40 & - & - \\
$3 / 4$ & 36.41 & $(0.28,0.29)$ & 54 \\
$4 / 5$ & 34.88 & $(0.20,0.22)$ & 43 \\
$5 / 6$ & 33.95 & $(0.16,0.18)$ & 36 \\
$6 / 7$ & 33.31 & $(0.13,0.15)$ & 32 \\
$3 / 5$ & 42.26 & $(0.34,0.38)$ & 11 \\
& & $(0.39,0.40)$ & 14 \\
$5 / 7$ & 37.62 & $(0.25,0.28)$ & 7.9 \\
& & $(0.25,0.37)$ & 32 \\
$7 / 9$ & \multirow{2}{*}{35.54} & $(0.17,0.25)$ & 25 \\
& & $(0.39,0.40)$ & 11 \\
$4 / 7$ & 43.65 & $(0.00,0.02)$ & 7.0 \\
& & $(0.05,0.06)$ & 9.3 \\
& & $(0.36,0.40)$ & 26 \\
$5 / 8$ & 41.12 & $(0.05,0.06)$ & 8.9 \\
& & $(0.29,0.40)$ & 32 \\
$7 / 10$ & \multirow{2}{*}{38.13} & $(0.05,0.06)$ & 7.8 \\
& & $(0.21,0.30)$ & 26 \\
\hline
\end{tabular}

Poincaré section $(y=0, \dot{y}<0)$ in the plane $e \cos \omega-e \sin \omega$. On the Poincaré section the periodic orbits are presented as fixed points while quasiperiodic orbits are projected as almost invariant curves due to the approximated integral $h_{z} \approx$ const. Such "invariant curves" may intersect each other.

The phase space topology changes essentially when the periodic orbit becomes unstable. In Fig. $7 \mathrm{~b}$ we present a Poincaré section of two trajectories starting close to the unstable fixed point of the periodic orbit of family $F_{a}^{4 / 5}$ at $e=0.202$ and $i=50^{\circ}$. We obtain that the orbits draw away and $\omega$ rotates. Generally, the orbits in this region of phase space are chaotic.

Extending our numerical exploration of families of periodic orbits in other resonances of first, second and third order we observe the existence of families having the same characteristics as those obtained for the 4/5,3/5 and 4/7 resonances. In Table 2 we present the regions in the eccentricity and the inclination domain where stable periodic orbits or, equivalently,

centers for ordered resonant motion can be found up to $e=0.4$. For all cases the stability regions start from $i_{\min }=0$ and extend up to a value $i_{\max }$ given in Table 2. In contrast, the eccentricity domain $\left(e_{\min }, e_{\max }\right)$ is narrow in most cases.

Our results are obtained in the framework of the circular RTBP and integrating the exact equations provided by (1). If we aim to associate these results with the real dynamics of the long-term evolution of trans-Neptunian objects, we should take into account the effect of the other major planets in the Solar System. Such additional perturbations may strongly affect the location and the width of the Kozai resonances. In a first approximation, the effects of other planets can be taken into account by adding their masses to the mass of the Sun or, equivalently, by reducing slightly the parameter $\mu$. We performed computations considering various values of the parameter $\mu\left(10^{-7} \leq \mu \leq 10^{-4}\right)$ and we found that the location of VCOs is practically unaffected. However, the model used by Morbidelli et al. (1995) and Nesvorný \& Roig (2001) possesses a Kozai resonance at lower eccentricity values. We showed that a $3 \mathrm{D}$ stable periodic solution is a sufficient condition for the existence of $\omega$-librating motion. We cannot make claims about the necessity of such a condition for a system of many degrees of freedom. The determination of the exact periodic orbits in a system of more than three degrees of freedom is a difficult task.

\section{The 3D elliptic problem}

When the planet is considered to revolve around the Sun on an elliptic orbit of eccentricity $e^{\prime}$ and period $T$, the system (1) is a non-autonomous system of three degrees of freedom with pe$\operatorname{riod} T=2 \pi$. Since exterior resonances are studied, i.e. the orbit of the massless body is outside the orbit of the planet, periodic orbits of the system should be of period $T=2 k \pi, k=1,2, \ldots$ A periodic orbit should satisfy the periodicity conditions (3) or (4) for a fixed value of $T$. Consequently, a periodic orbit of the circular problem should be a generating orbit for a family of periodic orbits in the elliptic problem if its period is a multiple of $2 \pi$.

A monoparametric family of symmetric periodic orbits is formed by analytic continuation with respect to the eccentricity of the planet $\left(e^{\prime}\right)$ which is the parameter of the family in this case. Such a family can be represented by a curve in the $4 \mathrm{D}$ space $x_{0}-\dot{y}_{0}-z_{0}-e^{\prime}$ or $x_{0}-\dot{y}_{0}-\dot{z}_{0}-e^{\prime}$ according to the symmetry of the periodic orbits. When $e^{\prime} \neq 0$, we get two possible configurations of the system at $t=0$. In the first configuration the planet is located at pericenter and in the second one at apocenter. Thus, from each generating orbit (or bifurcation point) two families bifurcate. Families of symmetric periodic orbits in the 3D elliptic RTBP were computed for the $1 / 2$ resonance by Kotoulas (2005).

Examining the variation of period $T$ along each family of the $3 \mathrm{D}$ circular problem at $4 / 5,3 / 5$ and $4 / 7$ resonances we found the bifurcation points summarized in Table 3 . The corresponding family where they belong is indicated. From each of these points two families of 3D periodic orbits in the elliptic problem are continued for $e^{\prime}>0$. All of them are linearly unstable. In Fig. 8 we present the families for the $4 / 5$ resonant case in the projection space $e^{\prime}-e_{0}$ (panel a) and $e^{\prime}-i_{0}$ (panel b), 

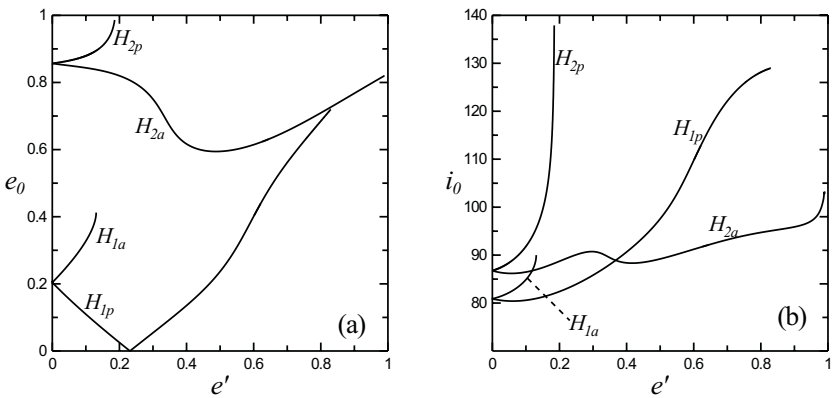

Fig. 8. Resonance 4/5: families of symmetric periodic orbits in the 3D elliptic RTBP.

Table 3. Bifurcation from the 3D circular to the 3D elliptic problem.

\begin{tabular}{cccc}
\hline \hline Resonance & Period & 3D Family & Bifurcation points \\
\hline $4 / 5$ & $10 \pi$ & $F_{a}$ & $e=0.204, i=80.9^{\circ}$ \\
$4 / 5$ & $10 \pi$ & $G_{a}$ & $e=0.865, i=86.8^{\circ}$ \\
\hline $3 / 5$ & $10 \pi$ & $\bar{F}_{a}$ & $e \approx 0.0, i=84.5^{\circ}$ \\
$3 / 5$ & $10 \pi$ & $F_{c 1}$ & $e=0.319, i=103.6^{\circ}$ \\
$3 / 5$ & $10 \pi$ & $F_{d}$ & $e=0.410, i=76.9^{\circ}$ \\
$3 / 5$ & $10 \pi$ & $F_{a}$ & $e=0.593, i=94.2^{\circ}$ \\
$3 / 5$ & $10 \pi$ & $\bar{G}_{a}$ & $e \approx 0.0, i=96.5^{\circ}$ \\
$3 / 5$ & $10 \pi$ & $G_{a}$ & $e=0.890, i=97.5^{\circ}$ \\
\hline $4 / 7$ & $14 \pi$ & $F_{a}$ & $e=0.112, i=87.0^{\circ}$ \\
$4 / 7$ & $14 \pi$ & $F_{b}$ & $e=0.097, i=92.6^{\circ}$ \\
$4 / 7$ & $14 \pi$ & $F_{b}$ & $e=0.383, i=82.7^{\circ}$ \\
$4 / 7$ & $14 \pi$ & $G_{a}$ & $e=0.896, i=88.3^{\circ}$ \\
\hline
\end{tabular}

where $e_{0}$ and $i_{0}$ denote the initial eccentricity and inclination of the massless body, respectively. From the first bifurcation point, given in Table 3 , we obtain the families $H_{1 p}$ and $H_{1 a}$, where $p$ or $a$ indicates the initial position of the planet (pericenter or apocenter, respectively). Correspondingly, we obtain the families $H_{2 p}$ and $H_{2 a}$ bifurcating from the second bifurcation point. The continuation of the family $H_{1 a}$ stops at $e^{\prime} \approx 0.13$ where the periodic orbits become very unstable. In contrast, family $H_{2 a}$ is continued almost up to the rectilinear case $e^{\prime}=1$.

Studying a particular planetary configuration, the periodic orbits should correspond to the particular value of $e^{\prime}$ and subsequently they are isolated. In the present study, the eccentricity of Neptune is small $\left(e^{\prime} \approx 0.01\right)$ and, subsequently, the corresponding periodic orbits should be located close to the bifurcation points. As is shown in Table 1, all bifurcation points are located at very high inclination values and therefore do not affect the phase space topology in the domain of low and moderate inclination values.

The effect of the eccentricity of Neptune is substantial on the evolution of the orbits that were periodic in the circular problem. In Fig. 9 we present the variation of the argument of perihelion along some trajectories associated with the stable periodic orbit of family $F_{a}^{4 / 5}$ at $i=10^{\circ}$, which has been discussed in Sect. 3.4. The four cases presented in this figure correspond to orbits with the same initial conditions as that of the periodic orbit except the argument of perihelion which is taken as $\omega_{0}=-90^{\circ}+\Delta \omega$. In panel a is $\Delta \omega=14^{\circ}$ and the
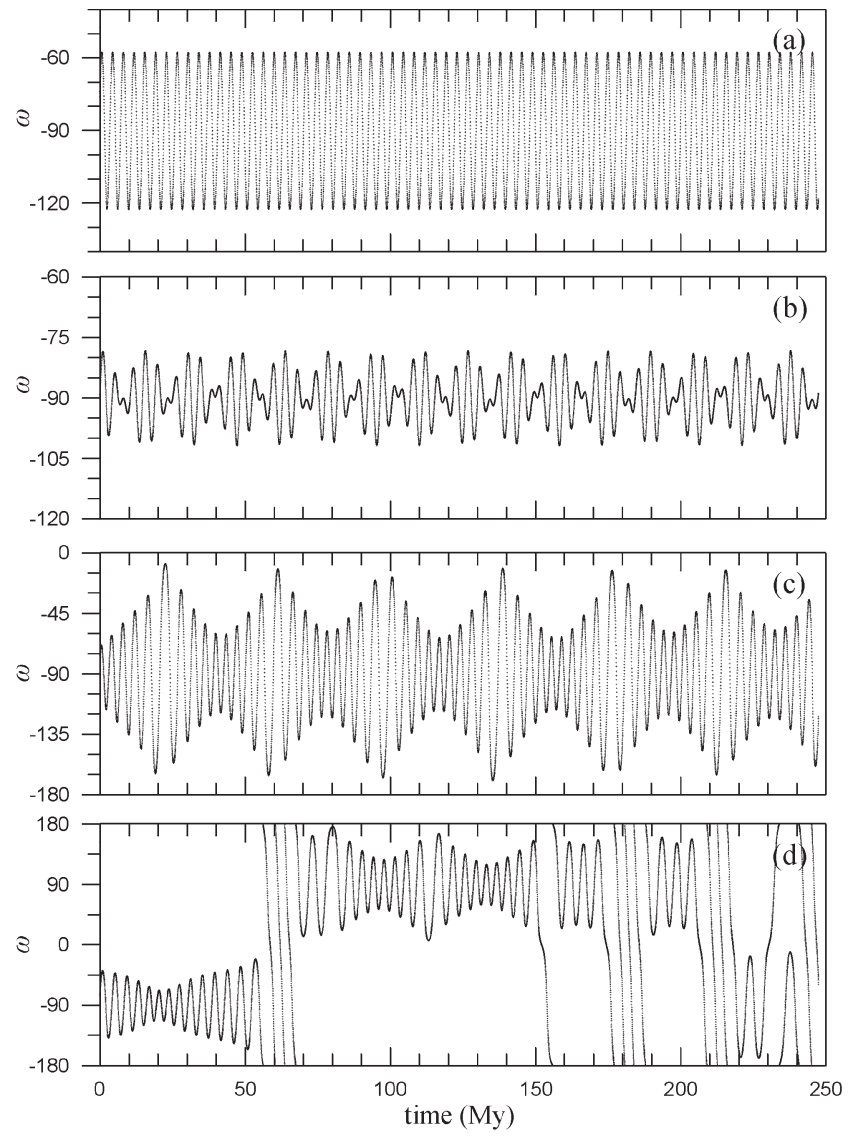

Fig. 9. The evolution of the argument of perihelion $(\omega)$ of the small body. The initial conditions correspond to a $4 / 5$ resonant stable periodic orbit of type $F$ with: $a_{0}=34.88 \mathrm{AU}, e_{0}=0.219, i_{0}=10^{\circ}$, $M_{0}=180^{\circ}, \Omega_{0}=-90^{\circ}$ and a) $\omega_{0}=-76^{\circ}, e_{N}=0$ (circular problem), b) $\omega_{0}=0^{\circ}, e_{N}=0.01, M_{N}=0, \varpi_{N}=0$ (elliptic problem), c) $\omega_{0}=-76^{\circ}, e_{N}=0.01, M_{N}=0, \varpi_{N}=180^{\circ}$, d) $\omega_{0}=-76^{\circ}$, $e_{N}=0.01, M_{N}=0, \varpi_{N}=0$.

Neptune is in a circular orbit. We obtain regular librations of $\omega$ since the orbit belongs to the Kozai resonance of the circular problem. Panel b corresponds to $\Delta \omega=0$, i.e. exactly to the periodic orbit of the circular problem, but Neptune is in an elliptic orbit. We observe that $\omega$ is forced to oscillate with a significant amplitude of about $12^{\circ}$. Thus, the stable periodic orbit of the circular system becomes quasi-periodic in the elliptical one. This is a typical situation for the stable periodic orbits of families $F$ and $G$ and, generally, is independent of the original position of Neptune, at least up to moderate eccentricity values of the small body's orbit. In panels $\mathrm{c}$ and $\mathrm{d}$ both the initial deviation $\Delta \omega=14^{\circ}$ and Neptune's eccentricity are taken into account. It is clear that the qualitative features of the long term evolution of the orbit depend on the initial position of Neptune.

\section{Conclusions}

In this paper we presented the results of a numerical exploration of periodic orbits in the exterior resonances with Neptune and in the framework of the spatial (3D) restricted three body problem. Our exploration extended in the whole 
domain of eccentricity and inclination values including retrograde orbits.

The 3D resonant periodic orbits form families generated from the vertically critical orbits of the planar problem. The continuation of the families showed that we have periodic orbits even for high inclination values. Indeed, we obtained families that either extend to planar retrograde orbits (i.e. $i=180^{\circ}$ ) or show a maximum in a domain of high inclination values. A classification of the families according to their continuation and the symmetry of orbits was presented in Sect. 2. The families generally continue smoothly and show a normal termination to planar VCOs (of direct or retrograde motion). Exceptional cases are the families $F_{c}$, which terminate at collision orbits with the Sun for $i \approx 90^{\circ}$.

A significant number of families of periodic orbits has been calculated for all resonances and the linear stability of orbits have been determined. The stable segments of the families denote the existence of Kozai resonances. The domain around periodic orbits of families $F$ and $G$ is associated with $\omega$-librating motion round the values $\pm 90^{\circ}$ and $0^{\circ}$ (or $180^{\circ}$ ), respectively. Such stable domains exist even for high eccentricity values. Families of type $F_{c}$, although they consist of highly eccentric orbits $(e>0.7)$, are stable. Families of type $G_{c}$ were not found.

When the eccentricity $e^{\prime}$ of the orbit of Neptune is taken into account in the model of the 3D-RTBP, periodic orbits form families by varying $e^{\prime}$. The families found in this case are unstable and exist only for high inclination values $\left(i>70^{\circ}\right)$. However, the $\omega$-librating motion is preserved around the periodic orbits of the circular problem, which, generally, become quasi-periodic under the effect of Neptune's eccentricity.

Acknowledgements. This work has been supported by the scientific programme "EPEAEK II, PYTHAGORAS", No. 21878, of the Greek Ministry of Education and E.U. The authors would like to thank Prof. J. D. Hadjidemetriou, who provided additional software to verify the results of the present work, and Dr. A. Morbidelli for his substantial reviewing remarks on the paper.

\section{References}

Beaugé, C., \& Ferraz-Mello, S. 1994, Icarus, 110, 239

Broucke, R. A. 1969, Periodic Orbits in the Elliptic restricted threebody problem, JPL Tech. Rep., 32

Broucke, R. A. 2001, Celest. Mech. Dyn. Astron., 81, 321

Celleti, A., Chessa, A., Hadjidemetriou, J., \& Valsecchi, G. B. 2002, Celest. Mech. Dyn. Astr., 83, 239

Gomes, R. S. 2003, Icarus, 161, 404

Gomes, R. S., Morbidelli, A., \& Levison, F. 2004, Icarus, 170, 492

Hahn, J. M., \& Malhotra, R., AJ, 117, 3041

Hadjidemetriou, J. D. 1988, Celest. Mech., 43, 371

Haghighipour, N., Couetdic, J., Varadi, F., \& Moore, W. B. 2003, ApJ, 596,1332

Hénon, M. 1969, A\&A, 1, 223

Hénon, M. 1973, A\&A, 28, 415

Kotoulas, T., \& Hadjidemetriou, J. D. 2002, Earth, Moon and Planets, 63

Kotoulas, T., \& Voyatzis, G. 2005a, Proc. IAU Coll., 197, 349

Kotoulas, T. 2005, A\&A, 429, 1107

Kuchner, M. J., Brown, M. E., \& Holman, M. 2002, AJ, 124, 1221

Michalodimitrakis, M. 1979, A\&A, 76, 6

Malhotra, R. 1996, AJ, 111, 516

Morbidelli, A. 1999, Cel. Mech. Dyn. Astr., 72, 129

Morbidelli, A. 2002, Modern Celestial Mechanics, Taylor and Francis

Morbidelli, A., Brown, M. E., \& Levison, H. F. 2003, Earth, Moon and Planets, 91, 63

Nesvorný, D., \& Roig, F., II 2001, Icarus, 150, 104

Papadakis, K. E. 2004, A\&A, 425, 1133

Szebehely, V. 1967, Theory of Orbits (Academic Press)

Varadi, F. 1999, AJ, 118, 2526

Voyatzis, G., \& Kotoulas, T. A. 2005b, Planetary and Space Science, 53, 1189

Yu, Q., \& Tremaine, S. 2001, AJ, 121, 1736

Zagouras, C., \& Markellos, V. V. 1977, A\&A, 59, 79 